PROCEEDINGS OF THE

AMERICAN MATHEMATICAL SOCIETY

Volume 128, Number 6, Pages 1605-1612

S 0002-9939(99)05171-0

Article electronically published on September 23, 1999

\title{
ON THE SCARCITY OF LATTICE-ORDERED MATRIX ALGEBRAS II
}

\author{
STUART A. STEINBERG
}

(Communicated by Ken Goodearl)

\begin{abstract}
We correct and complete Weinberg's classification of the latticeorders of the matrix ring $\mathbb{Q}_{2}$ and show that this classification holds for the matrix algebra $F_{2}$ where $F$ is any totally ordered field. In particular, the lattice-order of $F_{2}$ obtained by stipulating that a matrix is positive precisely when each of its entries is positive is, up to isomorphism, the only lattice-order of $F_{2}$ with $1>0$. It is also shown, assuming a certain maximum condition, that $\left(F^{+}\right)_{n}$ is essentially the only lattice-order of the algebra $F_{n}$ in which the identity element is positive.
\end{abstract}

\section{InTRODUCTION}

Let $F_{n}$ denote the $n \times n$ matrix ring over the ring $F$. If $\left(F, F^{+}\right)$is a latticeordered ring ( $\ell$-ring) with positive cone $F^{+}=\{\alpha \in F: \alpha \geq 0\}$, then $\left(F_{n},\left(F^{+}\right)_{n}\right)$ is also an $\ell$-ring. In [12] Weinberg has conjectured that if $F=\mathbb{Q}$ is the field of rational numbers and if $\left(\mathbb{Q}_{n}, P\right)$ is an $\ell$-ring with $1 \in P$, then $\left(\mathbb{Q}_{n}, P\right)$ is isomorphic to $\left(\mathbb{Q}_{n},\left(\mathbb{Q}^{+}\right)_{n}\right)$. He proved this conjecture for $n=2$ and also provided, for each $1<\beta \in \mathbb{Q}$, the following additional non-isomorphic lattice-orders $P_{\beta}$ of $\mathbb{Q}_{2}$ : there are idempotents $f_{1}, f_{2}, f_{3}, f_{4}$ in $\mathbb{Q}_{2}$ with $1=(1-\beta)\left(f_{1}+f_{2}\right)+\beta\left(f_{3}+f_{4}\right)$ and $\left(\mathbb{Q}_{2}, P_{\beta}\right)$ is the $\ell$-group direct sum of its totally ordered subrings $\mathbb{Q} f_{1}, \mathbb{Q} f_{2}, \mathbb{Q} f_{3}, \mathbb{Q} f_{4}$.

The assertion in [12] that each lattice-order of $\mathbb{Q}_{2}$ in which 1 is not positive is isomorphic to one of the $P_{\beta}$ 's is not quite correct, however. There is one additional lattice-order $P_{1}$ of $\mathbb{Q}_{2}$ that must be added to the list to make this statement correct. We will describe $P_{1}$ below.

If $R$ is an $\ell$-ring and an algebra over the totally ordered field $F$, then $R$ is an $\ell$-algebra over $F$ if it is a vector lattice, that is, if $F^{+} R^{+} \subseteq R^{+}$. We show that the description of the lattice-orders of $\mathbb{Q}_{2}$ that is given above also holds for the lattice-orders of $F_{2}$ which make it into an $\ell$-algebra over $F$ and that Weinberg's conjecture is true for $F_{n}$ provided that 1 has its maximum number $n$ of nonzero components in the decomposition of the $\ell$-algebra $\left(F_{n}, P\right)$ as a direct sum of totally ordered vector lattices over $F$.

Received by the editors March 27, 1998 and, in revised form, July 17, 1998.

1991 Mathematics Subject Classification. Primary 06F25; Secondary 15A48.

Key words and phrases. Lattice-ordered algebra, matrix algebra.

(C)2000 American Mathematical Society 


\section{Another lattice-Order for $\mathbb{Q}_{2}$}

We will first review a few definitions. A convex $\ell$-subgroup of an $\ell$-group is a subgroup $C$ that is a sublattice and is also convex: if $a \leq x \leq b$ with $a, b \in C$, then $x \in C$. An $\ell$-ideal of an $\ell$-ring is an ideal that is also a convex $\ell$-subgroup. A vector lattice over a totally ordered field $F$ is called archimedean over $F$ if it has no nonzero bounded subspaces.

If $R$ is a finite dimensional $\ell$-algebra over a totally ordered field $F$ and $R$ has no nonzero nilpotent $\ell$-ideals, that is, $R$ is $\ell$-semiprime, then $R$ is archimedean over $F$ by $[2$, Corollary 1, p. 51]. So it is a vector lattice direct sum of totally ordered $\ell$-simple subspaces (see [4, p. 3.27] or [8, Theorem 2.12]). Of course, if $F$ is a subfield of the real numbers $\mathbb{R}$, these totally ordered subspaces are embeddable in $\mathbb{R}$. Weinberg's method of proof for $\mathbb{Q}_{2}$ is to consider and eliminate all but two (actually, three) of the twenty-nine cases that arise depending on the number of summands, the dimensions of the summands, and the number and signs of the coordinates of 1 . The error occurs in the twenty-eighth case, which is case $(7 \mathrm{~d})$ of [12].

In (7d) we have that $\mathbb{Q}_{2}=E_{1} \oplus E_{2} \oplus E_{3} \oplus E_{4}$ as an -group where each $E_{i}$ is isomorphic to $\mathbb{Q}, 1=e_{1}+e_{2}+e_{3}$ where $0 \neq e_{i} \in E_{i}, e_{1}$ and $e_{2}$ have the same sign and it is opposite to that of $e_{3}$, and $0<n \in E_{4}$. The calculation down to the last sentence of (ii) is correct. So we have that $E_{1} \oplus E_{4}, E_{2} \oplus E_{4}$ and $E_{1} \oplus E_{2} \oplus E_{3}$ are subalgebras of $\mathbb{Q}_{2}$, and

$$
\begin{gathered}
e_{1}^{2}=k_{1} e_{1}, e_{2}^{2}=k_{2} e_{2}, k_{i} \in \mathbb{Q} \text { and } k_{1} k_{2}>0, n^{2}=n, \\
e_{2} n=e_{2}, e_{1} n=k_{1} n, n e_{2}=k_{2} n, n e_{1}=e_{1}, e_{1} e_{2}=0 .
\end{gathered}
$$

But $e_{1}$ and $e_{2}$ do not enter symmetrically in these calculations and so the assertion that $e_{2} e_{1}=0$ is not correct. We will complete the calculation and produce another lattice-order of $\mathbb{Q}_{2}$. Now, since $e_{2} e_{1}$ is in the subalgebra $E_{1} \oplus E_{2} \oplus E_{3}$,

$$
0 \leq e_{2} e_{1}=x e_{1}+y e_{2}+z e_{3} \text { with } x, y, z \in \mathbb{Q} \text { and } x e_{1}, y e_{2}, z e_{3} \geq 0 ;
$$

so

$$
0=e_{2} e_{1} e_{2}=y k_{2} e_{2}+z\left(1-e_{1}-e_{2}\right) e_{2}=\left(y k_{2}+z-z k_{2}\right) e_{2},
$$

and $y=\left(k_{2}-1\right) k_{2}^{-1} z$. We have that $e_{2} e_{1} \notin E_{1}$, since otherwise $\mathbb{Q}_{2} e_{1}=E_{1}$; so $z \neq 0$. Suppose that $e_{3}>0>e_{1}, e_{2}$. Then $z>0$ and $\left(k_{2}-1\right) k_{2}^{-1} z \leq 0$; but this is nonsense since $k_{2}<0$. So we must have that $e_{3}<0<e_{1}, e_{2}$, and hence $x \geq 0, z<0$ and $0<k_{2} \leq 1$. Since

$$
0=e_{1} e_{2} e_{1}=x k_{1} e_{1}+z e_{1} e_{3},
$$

$x=0$ and $e_{1} e_{3}=0 ;$ thus $e_{1}=e_{1}^{2}=k_{1} e_{1}$ and $k_{1}=1$. Also,

$$
0 \geq e_{3} e_{2}=\left(1-e_{1}-e_{2}\right) e_{2}=\left(1-k_{2}\right) e_{2} \geq 0
$$

gives that $k_{2}=1, e_{3} e_{2}=0, y=0$ and

$$
e_{1}=n e_{2} e_{1}=z n e_{3}=z n\left(1-e_{1}-e_{2}\right)=-z e_{1} ;
$$

hence, $e_{2} e_{1}=-e_{3}$. It is now easy to complete the following multiplication table:

\begin{tabular}{c|c|c|c|c|} 
& $e_{1}$ & $e_{2}$ & $e_{3}$ & $n$ \\
\hline$e_{1}$ & $e_{1}$ & 0 & 0 & $n$ \\
\hline$e_{2}$ & $-e_{3}$ & $e_{2}$ & $e_{3}$ & $e_{2}$ \\
\hline$e_{3}$ & $e_{3}$ & 0 & 0 & $-e_{2}$ \\
\hline$n$ & $e_{1}$ & $n$ & $-e_{1}$ & $n$ \\
\hline
\end{tabular}


The matrices

$$
e_{1}=\left(\begin{array}{rr}
0 & -1 \\
0 & 1
\end{array}\right), e_{2}=\left(\begin{array}{ll}
1 & 0 \\
0 & 0
\end{array}\right), e_{3}=\left(\begin{array}{ll}
0 & 1 \\
0 & 0
\end{array}\right), n=\left(\begin{array}{rr}
1 & 0 \\
-1 & 0
\end{array}\right)
$$

satisfy this table and the equation $1=e_{1}+e_{2}+e_{3}$. Thus, this lattice-order is given by $P_{1}=\mathbb{Q}^{+} e_{1}+\mathbb{Q}^{+} e_{2}+\mathbb{Q}^{+}\left(-e_{3}\right)+\mathbb{Q}^{+} n$, and, again, $\mathbb{Q}_{2}$ is the $\ell$-group direct sum of four totally ordered subrings.

\section{LATTICE-ORDERS OF $F_{2}$}

In this section we modify Weinberg's proof to obtain his theorem for any totally ordered field.

Theorem 1. Let $F$ be a totally ordered field.

(a) For each $1<\beta \in F$ there is a lattice-order $P_{\beta}$ of $F_{2}$ for which $R=$ $\left(F_{2}, P_{\beta}\right)$ is an $\ell$-algebra, and there are four idempotents $f_{1}, f_{2}, f_{3}, f_{4}$ in $P_{\beta}$ such that $R$ is the vector lattice direct sum of the subalgebras $F f_{1}, F f_{2}, F f_{3}, F f_{4}$ with $1=(1-\beta)\left(f_{1}+f_{2}\right)+\beta\left(f_{3}+f_{4}\right)$.

(b) There are idempotents $e_{1}, e_{2}$ and $n$ in $F_{2}$ and a nilpotent element $e_{3}$ such that $1=e_{1}+e_{2}+e_{3}$, and if $P_{1}$ is the positive cone of the vector lattice direct sum $F_{2}=F e_{1} \oplus F e_{2} \oplus F\left(-e_{3}\right) \oplus F n$, then $\left(F_{2}, P_{1}\right)$ is an $\ell$-algebra over $F$.

(c) If $R=\left(F_{2}, P\right)$ is an l-algebra over $F$, then $R$ is isomorphic to $\left(F_{2},\left(F^{+}\right)_{2}\right)$ if $1 \in P$, and, otherwise, $R$ is isomorphic to $\left(F_{2}, P_{\beta}\right)$ for exactly one $\beta \geq 1$.

Before giving the proof we will review some definitions and facts that we will need. If $M$ is a left module over the ring $D$, then $M$ is an $\ell$-module over $D$ if $D$ is a po-ring, $M$ is an $\ell$-group, and $D^{+} M^{+} \subseteq M^{+}$. The element $d \in D^{+}$is an $f$-element (respectively, a $d$-element) on $M$ if $d x \wedge y=0$ (respectively, $d x \wedge d y=0$ ) whenever $x$ and $y$ are elements of $M$ with $x \wedge y=0$. Each $f$-element is a $d$-element. The additive subgroup $T\left({ }_{D} M\right)$ of $D$ generated by the set of $f$-elements on $M$ is a convex directed subring of $D$. If $D$ is an $\ell$-ring, then $T\left({ }_{D} M\right)=\{d \in D:|d|$ is an $f$-element on $M\}$, and $T(D)=T\left({ }_{D} D\right) \cap T\left(D_{D}\right)$ is the convex $\ell$-subring of $f$-elements of $D$. $M$ is an $f$-module over $D$ if $D^{+} \subseteq T\left({ }_{D} M\right)$. For example, each vector lattice over a totally ordered division ring is an $f$-module. If $D$ is an $\ell$-ring, then $T(D)$ is an $f$-ring; that is, $T(D)$ is a right and left $f$-module over itself. If $D$ is a commutative po-ring and $R$ is an $\ell$-ring and an algebra over $D$, then $R$ is called an $\ell$-algebra if $R$ is an $f$-module over $D$.

Proof of Theorem 1. The proof of (a) is given by using the matrices $f_{1}, f_{2}, f_{3}$, and $f_{4}$ given below, and the proof of (b) is given by using the matrices presented in the previous section. As for (c), suppose that $P$ is a positive cone for $F_{2}$ such that $R=\left(F_{2}, P\right)$ is an $\ell$-algebra over $F$. As indicated at the beginning of section $2, R$ is the vector lattice direct sum of at most four totally ordered subspaces, and in [12], for $F=\mathbb{Q}$, Weinberg considers the various cases that arise depending on the number of summands, the dimensions of the summands and the coordinates of 1 . It is only the three cases $(1 \mathrm{c}),(7 \mathrm{~b})$ and $(7 \mathrm{~d})$ that lead to the lattice-orders $\left(\mathbb{Q}^{+}\right)_{2}, P_{\beta}$, and $P_{1}$ of $\mathbb{Q}_{2}$, respectively; in each of the other twenty-six cases it is shown that there is no lattice-order of the type under consideration. As can partially be seen from section 2 all of the arguments that are used in [12] for $\mathbb{Q}_{2}$ with the modification given in section 2 and some other minor modifications are valid for $R$ (although some of these arguments can be shortened) with the exception of those used in the three cases (3c), (3d), and (5). In each of these cases the fact that a totally ordered 
subspace of $\mathbb{Q}_{2}$ is embeddable in the complete field $\mathbb{R}$ is used to eliminate the possibility of a lattice-order of the type under consideration. We proceed to show that this fact can be avoided. Note that if $A$ and $B$ are totally ordered archimedean $F$-subspaces of an $\ell$-algebra and $C$ is a convex subspace, then $A B \subseteq C$ provided that $a b \in C$ for some $0 \neq a \in A$ and $0 \neq b \in B$.

Let $R=\left(F_{2}, P\right)$ be an $\ell$-algebra. In the three cases to be considered we have that $R=E_{1} \oplus E_{2}$ as vector lattices over $F$ where $E_{1}$ and $E_{2}$ are totally ordered subspaces.

(3c) Here we have $1 \in E_{1}$ and $\operatorname{dim}_{F} E_{2}=3$. Since $E_{2}$ is not an ideal of $R$, it cannot be a subring of $R$. So if $0 \neq f \in E_{2}$, then, by the Cayley-Hamilton theorem, $f^{2}=\alpha+\beta f$ with $\alpha, \beta \in F$ and $\alpha>0$. If $\beta=0$, then $E_{2}^{2} \subseteq E_{1}$, and this is impossible since $f E_{2}$ is 3 -dimensional over $F$. But then the trace function $\operatorname{tr}$ : $E_{2} \rightarrow F$ is monic.

(3d) In this case $\operatorname{dim}_{F} E_{i}=2$ and $0<1 \in E_{1}$. Then $E_{1}=T(R)$ is a division ring and hence is central by our extension of Tamhankar's extension of Albert's Theorem [10, Corollary 15]. This is absurd.

(5) Here, $1=e_{1}+e_{2}$ and $e_{1}<0<e_{2}$. Since $E_{i}$ is not an ideal it cannot be a subring of $R$. So if $0 \neq f \in E_{i}$, then $f^{2}=\alpha+\beta f$ with $\alpha \neq 0$. If $\beta=0$, then $f^{2}=\alpha e_{1}+\alpha e_{2} \geq 0$ implies that $\alpha=0$. Thus $\beta \neq 0$ and, again, $\operatorname{tr}$ is monic on $E_{i}$. This is impossible since $E_{1}$ or $E_{2}$ is at least 2-dimensional over $F$.

If $D$ is a totally ordered ring with the property that $D a D \neq 0$ if $0 \neq a \in D$, then it is easy to see that, for any $n,\left(D^{+}\right)_{n}$ is maximal among those ring partial orders $P$ of $D_{n}$ for which $D^{+} P+P D^{+} \subseteq P$; this is also the case if $n$ is infinite and $D_{n}$ is the ring of column finite matrices over $D$. None of the $P_{\beta}$ are maximal since in these lattice-orders 1 is not positive. Each $P_{\beta}$ is, however, contained in the cardinality of $F$ many lattice-orders each one of which is isomorphic to $\left(F^{+}\right)_{2}$. Specifically, let $P_{1}$ be given by the matrices in section 2 , and take $b, d \in F$ with $0 \leq b \leq-d$ and $d<0$. Then if $A_{b, d}=\left(\begin{array}{ll}1 & b \\ 0 & d\end{array}\right)$, it is easy to check that $A_{b, d}^{-1} P_{1} A_{b, d} \subseteq\left(F^{+}\right)_{2}$, and hence $P_{1}$ is contained in the lattice-order $X_{b, d}=A_{b, d}\left(F^{+}\right)_{2} A_{b, d}^{-1}$ of $F_{2}$. In particular, $P_{1} \subseteq X_{0,-1}=\left(\begin{array}{rr}F^{+} & -F^{+} \\ -F^{+} & F^{+}\end{array}\right)$. Also, $X_{b, d} \neq X_{b, h}$ if $d \neq h$ and $b>0$. This is a consequence of the fact that $C^{-1}\left(F^{+}\right)_{2} C=\left(F^{+}\right)_{2}$ with $\operatorname{det} C>0$ (respectively, det $C<0$ ) exactly when $C=\left(\begin{array}{ll}x & 0 \\ 0 & y\end{array}\right)$ (respectively, $C=\left(\begin{array}{ll}0 & x \\ y & 0\end{array}\right)$ ) and $x y>0$. So $A^{-1}\left(F^{+}\right)_{2} A=B^{-1}\left(F^{+}\right)_{2} B$ requires that the columns of $A$ are multiples of the columns of $B$ where the multipliers have the same sign. For $\beta>1$ let $P_{\beta}$ be given by the matrices from Weinberg's paper [12, p. 569]:

$$
\begin{array}{ll}
f_{1}=\left(\begin{array}{ll}
1 & 0 \\
0 & 0
\end{array}\right), & f_{2}=\left(\begin{array}{cc}
\beta(\beta-1)^{-1} & 1 \\
-\beta(\beta-1)^{-2} & -(\beta-1)^{-1}
\end{array}\right), \\
f_{3}=\left(\begin{array}{cc}
1 & (\beta-1) \beta^{-1} \\
0 & 0
\end{array}\right), & f_{4}=\left(\begin{array}{cc}
1 & 0 \\
(1-\beta)^{-1} & 0
\end{array}\right) .
\end{array}
$$

Then if $\beta(1-\beta)^{-1} \leq c \leq(1-\beta)^{-1}$ and $A_{c}=\left(\begin{array}{ll}1 & 1 \\ c & 0\end{array}\right)$, one can verify that $P_{\beta}$ is contained in the lattice-order $Y_{c}=A_{c}\left(F^{+}\right)_{2} A_{c}^{-1}$ of $F_{2}$, and $Y_{c} \neq Y_{t}$ if $c \neq t$. It can 
also be shown that if $1 \leq \beta, \delta \in F$ and $P_{\beta}$ and $P_{\delta}$ are the lattice-orders determined by the matrices listed above or in section 2 , then $P_{\beta} \subseteq P_{\delta}$ only if $\beta=\delta$.

If $F$ is a commutative totally ordered domain with totally ordered quotient field $Q$ and $R=\left(F_{n}, P\right)$ is an $\ell$-algebra over $F$, then $S=\left(Q_{n}, P^{e}\right)$ is an $\ell$-algebra over $Q$ and $R$ is an $F$ - $\ell$-subalgebra of $S$, where $P^{e}=\{x \in S: \exists 0<\alpha \in F$ with $\alpha x \in P\}$. Clearly, $T(S) \cap R=T(R)$ and $T(S) \neq 0$ if and only if $T(R) \neq 0$. It is easy to see, using Theorem 1, that for $n=2, T(S) \neq 0$ precisely when $S \cong\left(Q_{2},\left(Q^{+}\right)_{2}\right)$.

\section{4. $F_{n}$}

The element $0<s$ in the po-ring $D$ is a superunit if $s x \geq x$ and $x s \geq x$ for each $x \in D^{+}$. If $D$ is a totally ordered domain, then $D$ can be embedded in a (minimal) totally ordered unital domain $D_{u}$. Moreover, either $D$ has a superunit or $D \leq 1$ (see [7, Theorem III.3.5 and Chapter III] and [6, Theorem 1.6]). The module ${ }_{D} M$ is torsion-free if $d x=0$ implies that $d=0$ or $x=0$, for all $d \in D$ and $x \in M$. If ${ }_{D} M$ is a torsion-free $f$-module, then it is also a torsion-free $f$-module over $D_{u}$.

An $\ell$-ring is $\ell$-simple if it is not nilpotent and has no nonzero proper $\ell$-ideals. If a totally ordered domain $D$ is $\ell$-simple, then so is $D_{u}$ (and conversely); and $D$ has a superunit since if $0<\beta \in D$, then $\beta \leq \gamma \beta \gamma$ for some $\gamma \in D^{+}$and $1 \leq \gamma$ in $D_{u}$. Also, an $\ell$-simple $f$-ring has no proper nonzero one-sided $\ell$-ideals [7, IV.1.3 and IV.2.1] or [5, p. 132].

Let ${ }_{D} M$ be an $\ell$-module over the directed po-ring $D$. If $x$ and $y$ are elements of $M$, then $x$ is infinitely smaller than $y$ with respect to $D$ if $D|x| \leq|y|$; we indicate this relation by $x<<y . M$ is called archimedean over $D$ if whenever $x<<y$, then $x=0$. If $D x \neq 0$ for each $0 \neq x \in M$, then ${ }_{D} M$ is archimedean if and only if each bounded submodule of $M$ is trivially ordered.

Now, let $F$ be a commutative totally ordered domain with quotient field $Q$. If ${ }_{F} M$ is a torsion-free $f$-module, then its module of quotients (or injective hull) $E(M)=Q \otimes_{F} M$ is a vector lattice over $Q$ with positive cone $E^{+}=\{x \in E: \alpha x \in$ $M^{+}$for some $\left.0<\alpha \in F\right\}$. We are interested in deducing that ${ }_{F} M$ is archimedean when ${ }_{Q} E$ is archimedean. For this purpose we have

Lemma 2. Let $F$ be a commutative totally ordered domain with quotient field $Q$. The following statements are equivalent.

(a) $F$ is $\ell$-simple.

(b) If ${ }_{F} M$ is an $\ell$-module and $x<<y$ in $M$, then $x<<\alpha y$ for each nonzero $d$-element $\alpha$ on $M$; and $F$ has a superunit.

(c) If $x<<y$ in $F$, then $x<<\alpha y$ for each $0 \neq \alpha \in F$; and $F$ has a superunit.

(d) ${ }_{F} F$ is archimedean.

(e) There is an archimedean $f$-module over $F$ that is not torsion.

(f) If $C$ is a convex $\ell$-submodule of the torsion-free $f$-module ${ }_{F} M$, then $M / C$ is torsion-free.

(g) ${ }_{F} Q$ is archimedean.

(h) The following are equivalent for each torsion-free $f$-module ${ }_{F} M$ with module of quotients $E=E(M)$.

(i) ${ }_{F} M$ is archimedean.

(ii) ${ }_{F} E$ is archimedean.

(iii) ${ }_{Q} E$ is archimedean. 
Proof. First note that the implications (b) $\Rightarrow(\mathrm{c}), \quad(\mathrm{f}) \Rightarrow(\mathrm{a})$, and (h) $\Rightarrow(\mathrm{g}) \Rightarrow$ (d) $\Rightarrow(\mathrm{e})$ are obvious.

(a) $\Rightarrow$ (b). Suppose that $x<<y$ in the $\ell$-module $M$ and that $0<\alpha \in F$ is a $d$-element on $M$. If $\beta \in F^{+}$, there exists $\gamma \in F^{+}$with $\beta \leq \alpha \gamma$; so $\beta|x| \leq \alpha \gamma|x| \leq$ $\alpha|y|=|\alpha y|$.

(c) $\Rightarrow$ (d). If $0 \neq \alpha, \beta \in F^{+}$and $\alpha<<\beta$, then the convex subgroup $C(F \alpha)$ of $F$ generated by $F \alpha$ is a proper ideal of $F$. Consequently, $\alpha^{2}<<\alpha$, since if $\gamma \alpha^{2} \geq \alpha$ for some $\gamma \in F$, then $\gamma \alpha$ is a superunit of $F$ in $C(F \alpha)$. But then $\alpha^{2}<<\alpha^{2}$ and hence $F \leq 1$; this is impossible since $F$ has a superunit.

(e) $\Rightarrow$ (a). If $x$ is an element of the archimedean $f$-module ${ }_{F} M$ and $x$ is not torsion, then neither is $|x|$. Let $I$ be a proper $\ell$-ideal of $F$ and suppose that $0<\alpha \in I$ and $\beta \in F^{+} \backslash I$. Then $\alpha<<\beta$ and hence $\alpha x<<\beta x$; so we have the contradiction $\alpha x=0$.

(a) $\Rightarrow$ (f). Suppose that $0<\beta \in F, x \in M$ and $\beta x \in C$. If $\delta$ is a superunit of $F$, then $\delta \leq \gamma \beta$ for some $\gamma \in F$. So $|x| \leq \gamma \beta|x|=\gamma|\beta x| \in C$ and $M / C$ is torsion-free.

(a) and (d) $\Rightarrow(\mathrm{g})$. Let $\alpha, \beta, \gamma \in F^{+}$with $\alpha>0$ and suppose that $F \alpha^{-1} \beta \leq$ $\alpha^{-1} \gamma$. If $\rho \in F^{+}$, then $\rho \alpha \leq \alpha \delta$ for some $\delta \in F^{+}$. So $\rho \beta \leq \alpha \delta \alpha^{-1} \beta \leq \gamma$ and hence $\beta=0$.

(a) and (b) $\Rightarrow$ (h). For (i) $\Rightarrow$ (iii), if $Q\left|\alpha^{-1} x\right| \leq\left|\alpha^{-1} y\right|$ with $\alpha \in F^{+}$and $x, y \in M$, then $Q|x| \leq|y|$; hence $x=0$. For (iii) $\Rightarrow$ (ii), suppose that $F|x| \leq|y|$ where $x, y \in E$. Then for each $0<\alpha, \beta \in F, \beta|x| \leq \alpha|y|$; so $x<<y$ with respect to $Q$ and thus $x=0$. Clearly, (ii) $\Rightarrow$ (i).

From the proof just given it can be seen that Lemma 2 holds for other $f$-rings, also. For example, it holds if $F$ is any totally ordered left Öre domain, the equivalences (a) through (f) hold for any totally ordered domain, and (d) and (g) are equivalent if $Q$ is any quotient ring of the commutative $f$-ring $F$.

Recall that a module has finite Goldie dimension if it contains no infinite direct sum of nonzero submodules.

Lemma 3. Let $F$ be a commutative $\ell$-simple totally ordered domain, and let $R$ be an $\ell$-semiprime torsion-free $\ell$-algebra over $F$. Suppose that ${ }_{F} R$ has finite Goldie dimension. Then ${ }_{F} R$ is archimedean and is the direct sum of totally ordered $\ell$ simple submodules.

Proof. If $Q$ is the quotient field of $F$, then $S=Q \otimes_{F} R$ is a finite dimensional $\ell$-semiprime $\ell$-algebra over $Q$. So $S$ is archimedean over $Q$ and ${ }_{F} R$ is archimedean by Lemma 2 ; and, again, ${ }_{F} R$ is the direct sum of totally ordered submodules.

Let $x$ be an element of the $f$-module ${ }_{D} M$ over the directed po-ring $D$. A convex $\ell$-submodule of $M$ that is maximal with respect to not containing $x$ is called a $D$ value of $x$. For the theory of $D$-values see [3], [4] and [8]. We recall some facts about values. There is a bijection between the set of $D$-values of an element and its set of $\mathbb{Z}$-values. Also, if there is an integer $m$ such that each disjoint subset of nonzero elements of $M$ has cardinality at most $m$, then each element in $M$ has at most $m$ values. An element $x \in M$ has precisely $k$ values if and only if $x=x_{1}+\cdots+x_{k}$ where $\left\{x_{i}\right\}$ is a disjoint set and each $x_{i}$ has just one value. If $C$ is a convex $\ell$ submodule of $M$ and $x \in C$, then there is a bijection between its set of $D$-values in $C$ and its set of $D$-values in $M$. 
Theorem 4. Let $F$ be a commutative totally ordered domain. Suppose that $R=$ $\left(F_{n}, P\right)$ is an $\ell$-algebra with quotient $\ell$-algebra $S=\left(Q_{n}, P^{e}\right)$. The following statements are equivalent.

(a) $S$ is isomorphic to $\left(Q_{n},\left(Q^{+}\right)_{n}\right)$.

(b) $T(R)$ has an element with exactly $n$ values.

(c) $T(R)$ has an element with at least $n$ values.

(d) $1 \in P^{e}$ and 1 has at least $n$ values.

(e) $S$ contains an $F$ - $\ell$-subalgebra that is isomorphic to $\left(F_{n},\left(F^{+}\right)_{n}\right)$.

Proof. (a) $\Rightarrow(\mathrm{b}) . \quad T(S)$ is isomorphic to the $\ell$-subalgebra of diagonal matrices, so $1=e_{1}+\cdots+e_{n}$ where $\left\{e_{1}, \cdots, e_{n}\right\}$ are orthogonal primitive idempotents. If $0<\alpha \in F$ with $\alpha e_{i} \in T(R)$ for each $i$, then $\alpha=\alpha e_{1}+\cdots+\alpha e_{n}$ has exactly $n$ values.

(c) $\Rightarrow(\mathrm{d}) . T(S)$ is a finite dimensional $f$-subalgebra of ${ }_{Q} S$ and it is reduced [9, Lemma 2]. So $T(S)$ is the direct sum of totally ordered division algebras: $T(S)=D_{1} \oplus \cdots \oplus D_{k}$. Since $T(S)$ contains a set of $n$ disjoint elements, $k \geq n$. Now, if $e$ is the identity of $T(S)$, then $e=e_{1}+\cdots+e_{k}$ and $\left\{e_{i}\right\}$ is a set of orthogonal idempotents in $S$; so $k \leq n$ and $e=1$.

$(d) \Rightarrow($ a). Decompose $S$ as the vector lattice direct sum of totally ordered $\ell$-simple subspaces

$$
S=\bigoplus_{i=1}^{N} G_{i}
$$

As in the previous paragraph, $T(S)$ is the sum of $n$ of the $G_{i}, 1=e_{1}+\cdots+e_{n}$, and $\left\{e_{i}\right\}$ is a complete set of primitive disjoint orthogonal idempotents of $S$. For each $i, j, k, e_{i} G_{k}$ and $G_{k} e_{j}$ are convex subspaces of $G_{k}$; so for each $k$ there are unique $i$ and $j$ with $G_{k}=e_{i} G_{k} e_{j} \subseteq e_{i} S e_{j} \cong Q$. Thus, each $G_{k}=e_{i} S e_{j}$ is one-dimensional and $N=n^{2}$. We can now construct positive matrix units in the usual manner. For each $j$ with $2 \leq j \leq n$ the modules $e_{1} S$ and $e_{j} S$ are isomorphic, and so there exist elements $e_{1 j} \in e_{1} S^{+} e_{j}$ and $e_{j 1} \in e_{j} S^{+} e_{1}$ with $e_{1 j} e_{j 1}=e_{1}$ and $e_{j 1} e_{1 j}=e_{j}$. Let $e_{i i}=e_{i}$ and $e_{i j}=e_{i 1} e_{1 j}$. Then $\left\{e_{i j}: 1 \leq i, j \leq n\right\}$ is a set of positive matrix units in $S$ and $S=\bigoplus Q e_{i j} \cong\left(Q_{n},\left(Q^{+}\right)_{n}\right)$.

Since the equivalence of (a) and (e) and the implication (b) $\Rightarrow$ (c) are obvious, the proof is complete.

Recall that a unital ring is local if it has only one maximal right ideal.

Corollary 5. Let $F$ be a commutative unital $\ell$-simple totally ordered local domain. If $R=\left(F_{n}, P\right)$ is an $\ell$-algebra and $T(R)$ contains an element with at least $n$ values, then $R$ contains an $\ell$-subalgebra that is isomorphic to $\left(F_{n},\left(F^{+}\right)_{n}\right)$.

Proof. Let $S=\left(Q_{n}, P^{e}\right)$ be the quotient $\ell$-algebra of $R$. From Lemma 3 and the proof of Theorem 4

$$
R=\bigoplus E_{i j}, \quad S=\bigoplus G_{i j}, \quad 1 \leq i, j \leq n,
$$

where $E_{i j}$ is a totally ordered $F$ - $\ell$-simple submodule of $R$ and $E\left(E_{i j}\right)=G_{i j}$. Here, $1=e_{11}+\cdots+e_{n n}$ with $e_{i i} \in R$, and $e_{11} R \cong e_{i i} R$ for each $i$ since $F$ is local; so the positive matrix units $\left\{e_{i j}\right\}$ may be taken in $R$. Then $U=\bigoplus F e_{i j}$ is the desired $\ell$-subalgebra. 
If $1 \leq \beta \in Q$, then $\left(P_{\beta}\right)_{n}$ is a lattice-order of $Q_{2 n}$ in which 1 is not positive. Theorem 4 states that there is only one way (up to isomorphism) to lattice-order $S=Q_{n}$ so that 1 is positive and has its maximum number of nonzero components in the decomposition of $S$ into totally ordered subspaces. Let us note that the obstruction to removing this latter condition comes from our inability to eliminate 1 having its minimum number of nonzero components. Specifically, we note that Weinberg's Conjecture can be rephrased as follows.

The following statements are equivalent for the totally ordered field $Q$.

(W1) $\forall n \geq 1$, if $\left(Q_{n}, P\right)$ is an $\ell$-algebra with $1 \in P$, then $\left(Q_{n}, P\right) \cong\left(Q_{n},\left(Q^{+}\right)_{n}\right)$.

(W2) $\forall n \geq 2, Q_{n}$ has no algebra lattice-orders for which $T\left(Q_{n}\right)=Q 1$.

(W3) $\forall n \geq 2, Q_{n}$ has no algebra lattice-orders with $1 \in T\left(Q_{n}\right)$ and $T\left(Q_{n}\right)$ is totally ordered.

The implications (W1) $\Rightarrow(\mathrm{W} 3) \Rightarrow(\mathrm{W} 2)$ are obvious. For (W2) $\Rightarrow(\mathrm{W} 1)$, suppose that $S=\left(Q_{n}, P\right)$ is an $\ell$-algebra with $1 \in P$. Then $1=e_{1}+\cdots+e_{m}$ where $\left\{e_{i}\right\}$ is a complete set of orthogonal idempotents in $T(S)$. Now, $m \geq 2$ since if $m=1$, then $T(S)$ would be central by [10, Corollary 15], and hence $T(S)=Q 1$. If some $e_{i}$ is not primitive in $S$, then $e_{i} S e_{i} \cong Q_{t}$ with $2 \leq t<n$, and $e_{i} S e_{i}$ is a convex $\ell$-subalgebra of $S$. But then $e_{i}=f_{1}+\cdots+f_{k}$ with $2 \leq k$ and $\left\{f_{j}\right\}$ is a set of nonzero orthogonal idempotents in $e_{i} S e_{i} \cap T(S)$; and this contradicts the completeness of $\left\{e_{i}\right\}$.

\section{REFERENCES}

[1] A. A. Albert, On ordered algebras, Bull. Am. Math. Soc. 46 (1940), 521-522. MR 1:328e

[2] G. Birkhoff and R. S. Pierce, Lattice-ordered rings, An. Acad. Brasil 28 (1956), 41-69. MR 18:191d

[3] P. Conrad, The lattice of all convex $\ell$-subgroups of a lattice-ordered group, Czechoslovak Math. J. 15 (1965), 101-123. MR 30:3926

[4] P. Conrad, Lattice-ordered groups, Tulane Lecture Notes, Tulane University, 1970.

[5] L. Fuchs, Partially ordered algebraic systems, Akademia Kiadó, Budapest, 1963. MR 30:2090

[6] M. Henriksen and J.R. Isbell, Lattice-ordered rings and function rings, Pacific J. Math. 12 (1962), 533-565. MR 27:3670

[7] D. G. Johnson, A structure theory for a class of lattice-ordered rings, Acta. Math. 104 (1960), 163-215. MR 23:A2447

[8] S. A. Steinberg, Finitely-valued f-modules, Pacific J. Math. 40 (1972), 723-737. MR 46:5205

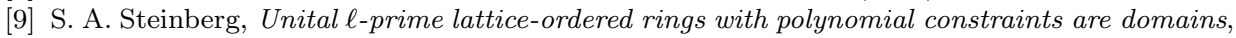
Trans. Amer. Math. Soc. 276 (1983), 145-164. MR 84d:16050

[10] S. A. Steinberg, Central f-elements in lattice-ordered algebras, Ordered Algebraic Structures, Eds. J. Martinez and C. Holland, Kluwer (1993), 203-223. MR 96d:06023

[11] M. V. Tamhankar, On algebraic extensions of subrings in an ordered ring, Algebra Universalis 14 (1982), 25-35. MR 83f:06029

[12] E. C. Weinberg, On the scarcity of lattice-ordered matrix rings, Pacific J. Math. 19 (1966), 561-571. MR 34:2635

Department of Mathematics, The University of Toledo, Toledo, Ohio 43606-3390

E-mail address: ssteinb@uoft02.utoledo.edu 